\title{
Géolinguistique
}

$20 \mid 2020$

Varia

\section{*(EX-)TABANUS : une matrice sémantique neurocognitive pour des taons prototypes des esprits errants dans les Alpes francoprovençales et occitanes}

*(EX-)TABANUS: A Neurocognitive Semantic Matrix for Horseflies as Prototypes of Wandering Spirits in Francoprovençal and Occitan Alps

\section{Fabio Armand}

\section{(2) OpenEdition}

\section{Journals}

Édition électronique

URL : http://journals.openedition.org/geolinguistique/2102

DOI : 10.4000/geolinguistique.2102

ISSN : $2650-8176$

Éditeur

UGA Éditions/Université Grenoble Alpes

Édition imprimée

ISBN : 978-2-37747-246-8

ISSN : 0761-9081

\section{Référence électronique}

Fabio Armand, «*(EX-)TABANUs : une matrice sémantique neurocognitive pour des taons prototypes des esprits errants dans les Alpes francoprovençales et occitanes », Géolinguistique [En ligne], 20 | 2020, mis en ligne le 01 décembre 2020, consulté le 19 janvier 2021. URL : http://journals.openedition.org/ geolinguistique/2102; DOI : https://doi.org/10.4000/geolinguistique.2102

Ce document a été généré automatiquement le 19 janvier 2021.

Géolinguistique 


\title{
*(EX-)TABANUS : une matrice
} sémantique neurocognitive pour des taons prototypes des esprits errants dans les Alpes francoprovençales et occitanes

\author{
*(EX-)TABANUs: A Neurocognitive Semantic Matrix for Horseflies as Prototypes of \\ Wandering Spirits in Francoprovençal and Occitan Alps
}

Fabio Armand

\section{Remerciements}

Nous voulons remercier Christian Abry, ami et collaborateur de longue date, pour nous avoir accompagné dans les réflexions présentes dans cette contribution et dans l'élaboration de notre perspective de sémantique neurocognitive expérientielle.

\section{Quand la folkloristique de la narration ouvre la voie à un modèle de sémantique cognitive...}

Il y avait à Cretaz un vieil homme qui, racontait-on, une fois par mois, le jeudi soir, ne laissait entrer personne chez lui pour la veillée : ce vieil homme s'enfermait chez lui, il s'étendait sur un grand banc et s'endormait aussitôt; une fois endormi un grand taon noir sortait de sa bouche et l'homme restait comme mort. Il restait ainsi pendant deux ou trois heures et on disait que son esprit allait à la senagogga [sabbat des sorciers]. [...] Après deux ou trois heures, cette " chose » se terminait: le vieil homme revenait à lui et se réveillait à nouveau [...] ceux qui un jour avait su cela l'espionnèrent et, avant qu'il ne revienne à lui (mot-à-mot «comme en vie »), ils virent rentrer dans la bouche le gros taon. (Notre traduction française de la version patoise publiée dans Guichardaz \& Fassò, 1974 : 161-162.) 
1 Ce récit de croyance, collecté dans la vallée de Cogne (Vallée d'Aoste, Italie), représente le point de départ d'une traversée en narration des Alpes francophones, dominées par le mont Blanc, afin de mettre en évidence une possible matrice linguistique qui nous permettra d'encadrer les déplacements aériens hors-du-corps, comme celui effectué par ce vieil homme de Cretaz, dans un modèle sémantique plus général. Ce document narratif relatant le voyage sous forme de taon-âme d'un sorcier de la vallée de Cogne a des parallèles importants sur les autres versants alpins. C'est ce que nous offre la collecte alpine réalisée par Charles Joisten (2005-2010), récemment éditée dans son intégralité par Alice Joisten et Nicolas Abry. Cet énorme trésor de contes et de récits de croyance représente la base fondamentale pour aborder l'ethnographie de la narration en domaine alpin. La totalité des narrations a été classée en utilisant le Motif-Index of Folk-Literature de Stith Thompson (1966), œuvre monumentale pour toute enquête comparative dans le domaine de la folkloristique narrative. Un document isérois collecté par Charles Joisten nous rapporte que :

[Une femme] piochait des pommes de terre au Chastera. Après le repas de midi, elle se couche à l'ombre et s'endort. Une grosse mouche sort de sa bouche et s'envole dans la direction du Villard-Saint-Jean. Au bout de quelques minutes, tant elle allait vite, elle atteint ce village, pénètre dans une maison, enfonce une aiguille dans la tête d'un enfant qui dormait. Là-bas, la sorcière repose toujours. Passe un garçon qui, pour s'amuser, la tourne, face contre terre. La mouche revient de son expédition, mais ne peut réintégrer sa demeure. [...] Les sœurs de la femme arrivent à cet instant et la retournent, face contre ciel. On lui demande des explications. «Je viens, dit-elle, du Villard-Saint-Jean où j'ai enfoncé une aiguille dans la tête d'un enfant." On veut la faire repartir immédiatement. "Je ne le peux pas avant demain. » Le lendemain, elle retourne dare-dare au village. Mais il était trop tard. L'enfant était mort. (2005: 198-199)

2 Pour traquer ce type de narrations dans les Alpes, nous nous tournons enfin vers les vallées vaudoises du Piémont, où nous retrouvons des actions malfaisantes accomplies par des sorciers en forme de taon ou autres insectes bourdonnants. Jalla (1926:38), dans son importante collecte de légendes, nous rapporte l'expérience d'une femme, servante en France, qui s'endort : «[...] on voit un bourdon sortir de sa bouche. On lui fait avouer que ce $\operatorname{masc}^{1}$ [sorcière] est allé planter une épingle dans la tête de l'enfant du maire de son pays.» Quatre autres versions du récit "L'insecte sorcier» sont présentées dans le recueil Tradizioni orali delle Valli valdesi del Piemonte par Marie Bonnet (1994 [1910-1914] : 310-314). Nous proposons, à titre d'exemple, la version suivante ${ }^{2}$, collectée à Baisse de Maneille auprès d'Honorine Tron :

Une jeune fille de Pomaret se laissa séduire par un sorcier ; mais, après quelques temps, elle préféra les avances d'un jeune homme plus sympathique, et se fiança avec lui. L'amoureux délaissé, fou de jalousie, jura de se venger aux dépens de son rival. On raconte qu'il s'endormait avec la bouche ouverte; alors, le malin esprit qu'il servait sortait d'entre ses lèvres sous la forme d'un taon, et allait battre la campagne toute la nuit, en faisant du mal au malheureux fiancé. L'insecte pouvait, selon son bon plaisir, prendre toutes les formes qu'il voulait, y compris celle humaine : il savait aussi le secret de se rendre invisible. Vers l'aube, le taon rentrait dans la bouche du sorcier, qui avait toujours dormi ; à ce moment, le jeune homme se réveillait. (Ibid. : 311-312)

Des bourdons, des grosses mouches et des taons... Transculturellement, les insectes diptères représentent de bonnes métaphores pour rendre compte du domaine du sommeil et du rêve (Motte Florac \& Thomas, 2003). En ce qui concerne les expériences hors-du-corps, ces petits insectes bourdonnants transalpins représentent l'âme-esprit 
du dormeur, sortant du corps physique resté immobile pour réaliser des vagabondages nocturnes. La série de documents narratifs que nous venons de présenter pour les voyages extatiques de ces sorciers alpins nous renvoie aux motifs narratifs génériques ${ }^{3}$ identifiés par Thompson (1966) dans son Motif-Index of Folk-literature: E721 - Soul journeys from the body; E730 - Soul in animal form et, plus en particulier, E715.3Separable soul in insect. Pour les motifs de type sorcellaire, nous indiquons aussi G211.5.1 - Witch in form of fly et, le plus spécifique, G251.1 - Witch recognized by seeing wasp (beetle) enter her mouth while asleep. Only when it enters she can be awakened.

\section{Insectes diptères et expériences hors-du-corps : une approche d'anthropologie neurocognitive}

4 Ces observations dans le domaine de la folkloristique de la narration nous offrent des indices expérientiels pour la compréhension du substrat neurocognitif qui se trouve à la base de ces narrations alpines. L'aspect qui nous intéresse tout particulièrement dans les versions que nous venons de rapporter concerne fondamentalement le processus de sortie du corps de ces sorciers alpins, que ce soit sous la forme de taon, de bourdon, de mouche ou de papillon. L'opposition évidente entre le dynamisme de l'âme en vagabondage et l'immobilité de l'enveloppe corporelle vidée de l'esprit qui l'anime relatent un processus de métensomatose de l'âme en insecte. Ces documents nous permettent alors de mettre en évidence la figure du taon, prototype de la grosse mouche ou, plus généralement, des insectes diptères, dans un processus de narrativisation des expériences hors-du-corps vécues par les sujets protagonistes de ces récits.

5 Afin d'appréhender les bases neurocognitives des expériences hors-du-corps, nous insérons plus explicitement ces réflexions dans le cadre du modèle transculturel d'anthropologie neurocognitive BRAINCUBUS (Cathiard \& Armand, 2014; Armand, Cathiard \& Abry, 2019), qui nous permet de rattacher ces expériences à une des composantes d'un état dissocié du cerveau dit paralysie du sommeil. Ce quatrième état du cerveau humain a une haute prévalence, ce qui permet à $40-50 \%$ de la population d'en faire l'expérience au moins une fois au cours de la vie. Au cours de cette expérience, les grands muscles striés du sujet sont paralysés, comme dans la phase de sommeil paradoxal, à l'exception aussi des muscles oculaires et de muscles des lèvres (houppe du menton). Toutefois, ses entrées sensorielles restent intactes, comme dans l'état de l'éveil, ce qui permet une perception vivide de l'environnement réel. Au cours de cette expérience, le sujet ressent avant tout une sensation d'immobilisation forcée (1) : son corps ne répond plus à ses intentions motrices et il ressent sur lui un poids; il ne peut ni bouger, ni parler et il perd aussi le contrôle volontaire de sa respiration. En outre, le sujet peut percevoir son environnement réel et (2) il y perçoit aussi une présence indéniable (3): cette perception peut être visuelle, mais elle peut aussi être accompagnée de sensations auditives, olfactives ou tactiles. Cette présence est pour lui terrifiante, plus rarement érotique (4) (Abry \& Joisten, 2002 : 140-141). Les personnes qui ont vécu cette expérience rapportent souvent d'avoir vécu des expériences horsdu-corps, se sentant flotter dans l'air en regardant de l'extérieur leur propre corps physique resté immobile.

6 En proposant une approche "experience-centered", qui reconnaît en même temps l'expérience narrativisée par le sujet et la réalité neurophysiologique de telle 
expérience, nous avons pu rattacher au phénomène de l'âme partant visiter sous forme de taon-esprit une expérimentation de stimulation corticale préopératoire en épileptologie, réalisée par l'équipe dirigée par le neurologue Olaf Blanke, afin d'en déceler les bases neurales. En 2002, en stimulant corticalement la jonction temporopariétale droite d'une femme épileptique, l'équipe de Blanke a déclenché chez la patiente une expérience hors-du-corps (OBE), expérience qu'elle n'avait jamais vécue avant au cours de ses attaques épileptiques. Elle a rapporté se sentir flotter dans l'air se soulevant vers le plafond d'où elle pouvait regarder en bas son propre corps physique gisant en position supine. Nous assistons ainsi à une dissociation entre le corps physique et le corps somato-sensoriel : ce qui génère un corps "fantôme » auto-agentif, habité par le Self, qui regarde d'une perspective extra-personnelle le corps physique du sujet.

\section{Bases dialectologiques pour une sémantique neurocognitive des formes * (EX-) TABANUS}

7 Pour pouvoir avancer dans la construction de notre sémantique neurocognitive des expériences hors-du-corps, nous avons présenté les fondements neuraux sur lesquels reposent les narrations relatant les vagabondages nocturnes sous forme d'âme-insecte. Comme nous l'avons annoncé, nous réservons au taon une position privilégiée dans notre analyse, en le considérant comme le prototype de la grosse mouche et de tout autre type d'insecte et de vermines nuisibles. Une approche dialectologique, intégrée dans le modèle d'anthropologie neurocognitive que nous avons brièvement énoncé, nous fournit les éléments pour faire le pont entre les insectes diptères présents dans nos récits de croyance alpins et l'expérience de paralysie du sommeil à la base de ces déplacements extatiques.

\subsection{Tavané dans les parlers francoprovençaux valdôtains}

8 L'attention particulière que nous avons attribuée au gros taon noir de Cogne et au bourdon des vallées vaudoises est en rapport avec une référence précise présente dans les réponses données pour la question "cauchemar» de l'Atlas des patois valdôtains. À côté de beur sondzo, « mauvais rêve ", le point d'Arnad atteste une forme tavan-a pour indiquer le cauchemar. Une autre attestation similaire, se rapportant cette fois-ci au domaine du rêve et du sommeil, est présente dans le Dictionnaire du patois valdôtain de l'abbé Jean-Baptiste Cerlogne (1907:282), où nous retrouvons la forme verbale tavané pour « rêver, divaguer, perdre son temps ci et là ». Le Nouveau dictionnaire du patois valdôtain (Chenal \& Vautherin, 1997: 1677-1678), de structure indéniablement cerlognenne (cf. Armand \& Dunoyer, 2019), reprend ces traductions, « rêver, divaguer, perdre son temps, flâner ", mais y ajoute aussi, sous une autre entrée, la signification d'«assommer ». Plus récemment, le Dizionario del dialetto francoprovenzale di Hône (Gruppo «Amis du patois», 2007:339) nous propose le verbe tavanéi avec la signification de "vaneggiare, fantasticare, farneticare; parlare nel sonno; sproloquiare, straparlare, sragionare, dare i numeri » : nous retrouvons encore une fois une référence au domaine du sommeil dans le phénomène de la somniloquie qui survient au cours de la phase du sommeil léger. 
Enfin, la consultation de deux autres ouvrages locaux nous permettent de mieux cerner ces champs sémantiques. Le Dictionnaire du patois de Champorcher (Glarey, 2011 : 328) nous rapporte pour tavané, "dire des sottises, délirer », en nous proposant l'exemple "lou maladou y a tavanà totta la nóit a cóza què avéive la tsivra [le malade a déliré toute la nuit à cause qu'il avait la fièvre] ", et il ajoute le substantif tavanéiri pour " histoire sans sens ; sottise " (ibid. : 329). Le Dictionnaire du patois de Chambave (Lavoyer, $2017: 288$ ) inclut aussi le verbe tavanèi, «flâner, perdre son temps ; ne pas savoir quoi faire ».

\subsection{De l'autre côté des Alpes : les parlers francoprovençaux de France et de la Suisse romande}

10 À côté de ces tavané, dérivés du latin TABANUS (FEW 13, 1,2), des patois francoprovençaux de la Vallée d'Aoste, nous trouvons une autre série verbale formée du composé * EX- + TABANUS. Le Glossaire des patois francoprovençaux (Duraffour, 1969), nous rapporte l'adjectif étàvàni (3368) attesté à Viriat (point d'enquête 31, département de l'Ain), avec la signification de "inerte, engourdi de froid ou assommé par un coup ». En consultant la carte 1028, "(J'ai failli) m'évanouir", de l'Atlas linguistique et ethnographique du Lyonnais (ALLy), nous pouvons individualiser une petite aire sudoccidentale ${ }^{4}$, à l'extrémité d'une aire plus vaste s'étendant sur le Massif central jusqu'au Languedoc (voir ALMC, carte 1594; ALF, carte 1561), occupée par le type lexical étavañi.

11 En outre, le Glossaire des patois de la Suisse romande (GPSR 6, 795) atteste le type verbal ètavani avec la signification transitive de " assommer, étourdir en donnant un coup » et celle intransitive de "s'évanouir, tomber en syncope»; dans le Vaud, une nouvelle signification, « tuer », est aussi attestée dans la forme èl ètavani, « il est mort ».

\subsection{Observations occitanes}

Enfin, comme nous l'avons déjà noté, nous trouvons des attestations du composé de *EX- + TABANUS dans les parlers occitans (cf. apr. estavanir). Le Trésor dòu Felibrige (1878: 1055) nous donne un estavani, estabani, astabani avec les significations de "tomber en faiblesse, pâmer, perdre la respiration à force de pleurer, comme il arrive aux enfants ». De même, le Dictionnaire occitan de communication, dirigé par Arve Cassignac (disponible sur <www.panoccitan.org>), nous propose pour ( $s^{\prime}$ )estavanir les significations de "s'évanouir» et «se pâmer". Le Diccionari general occitan. A partir dels parlars lengadocians $\left(2002^{5}\right)$, premier dictionnaire monolingue occitan, à base de languedocien, réalisé par Louis Combes, alias Joan de Cantalausa, nous rapporte, pour la forme estavanir, "assucar (far pèrdre coneissença d'un brave còp sul cap); afalhocar » et, pour la forme pronominale, s'estavanir, "pèrdre coneissença ». Enfin, le Dictionnaire étymologique de la langue d'oc ${ }^{6}$ nous vient en aide pour déceler la complexe sémantique de ce type lexical, en nous offrant l'accès, sous l'article tavan, à une intéressante réflexion conduite par Robert Geuljans autour de ce terme : «le verbe composé ${ }^{*} e x^{-}+$tabanus : $\left(s^{\prime}\right)$ estavani « s'évanouir, se pâmer » limité au frpr. [francoprovençal] et l'occitan a dû suivre l'évolution sémantique suivante: «s'inquiéter » $>$ «troubler » > «troubler la raison $»>$ " perdre connaissance $»^{7}$. 


\section{Passage en inalpage pour une matrice sémantique pastorale : tabanus, asilus et oestrus}

13 La perspective dialectologique que nous avons esquissée dans la section précédente nous a permis de mettre en évidence deux types lexicaux principaux, les deux formés sur la base latine TABANUS, « taon », avec ou sans la présence du préfixe EX-, marquant la séparation. En partant des formes verbales simples issues de TABANus, le Französisches etymologisches Wörterbuch (FEW 13, 1,2) nous propose des indications précises rattachées au champ sémantique de l'errance : en partant des sens «bourdonner (en parlant des mouches) » et « fredonner », nous passons aux sens figurés de " tournoyer à la manière des bourdons ", "rôder ça et là ", « errer ", "voltiger à l'aventure ça et là sans dessein », «s'agiter dans le vide sans but préconçu », etc. Nous trouvons aussi, pour Tarascon, un autre emploi figuré, « papillonner autour des jeunes filles, ou faire la coquette devant les garçons", pour aboutir carrément aux sens plus forts de « importuner, ennuyer, déranger, agacer ».

Cette dérivation sémantique nous semble donc amener de la plus faible inquiétude vers la connotation plus forte du trouble, liée au fait de tourmenter et harceler. En effet, cet insecte diptère et suceur représente une sorte de grosse mouche trapue dont la femelle, pendant la saison estivale, tourmente de ses piqûres les bœufs, les autres animaux domestiques et, bien sûr, l'homme. Ces considérations étymologiques nous permettent ainsi de tisser le lien avec l'action des taons qui tourmentent le bétail et les hommes. En particulier, nous suivrons un parcours qui, en nous plongeant dans l'univers de l'élevage ancien, nous fait découvrir les développements de deux mots latins qui se réfèrent aux insectes qui tourmentent le bétail : le tabanus ou asilus (Tabanus bovinus) et le oestrus (Oestrus ovis). Ces deux termes indiquent communément une catégorie d'insectes qui sucent le sang des animaux qui paissent dans les hauts pâturages de montagne. Comme le note Alinei :

[...] avec un passage tout à fait logique, que les dictionnaires étymologiques ne rapportent pas (et pour cela je me propose de revenir ailleurs), seul le nom latin du premier, asilus, est parvenu à signifier (en italien ; pour les dialectes voir ci-dessous) la constante mais inoffensive gêne causée par l'insecte qui, précisément, « harcelle » [assilla, en italien] l'animal. Alors que le second, l'oestrus, est parvenu à juste titre à désigner, déjà en latin, l'effet pathologique sur le système nerveux de l'animal dont le cerveau a été atteint par les larves de l'insecte, et de là, la fureur guerrière, le délire prophétique, l'inspiration poétique, la fureur passionnée, préfigurés par le comportement insensé de l'animal. (2009: 16 ; notre traduction)

En réfléchissant plus précisément autour du terme asilus qui, indiquant originellement le nom de cet insecte tourmenteur de bétail, a servi ensuite à désigner les effets sur les animaux (FEW 1, 153; 25, 433-434), nous trouvons, pour les parlers francoprovençaux de la Vallée d'Aoste, les termes bedzolé (Cerlogne, 1907 : 91 ; Chenal \& Vautherin, 1997 : 188), désoulé (Chenal \& Vautherin, 1997 : 516) et, pour Champorcher, adzoulé (Glarey, 2011 : 51) pour indiquer l'action des vaches qui s'enfuient du pâturage, la queue en l'air, à cause de la chaleur ou dérangées par ce tabanus/asilus qui les tourmente. Ces termes seraient une dérivation de la forme *asilare, "prendre la mouche ", dont asilus, le taon, duquel dériverait aussi le mot italien assillo, hantise, inquiétude (Lessico etimologico italiano, III-1: 1587). Nous pouvons ainsi mettre en évidence une première matrice sémantique pastorale qui montre la fonction de harceleur du bétail réalisée par ce tabanus/asilus dans les hauts pâturages alpins : cette même matrice se retrouve dans les 
séries verbales issues du latin TABANUS soulignant l'errance nuisible et importune de ces insectes diptères, voire le harcèlement malfaisant accompli par les sorciers-taons des Alpes lors de leurs vagabondages nocturnes.

\section{Taons et esprits en vagabondage : une matrice sémantique pour les expériences hors-du-corps}

16 Pour revenir aux documents narratifs qui ont ouvert cette contribution concernant les voyages extatiques des sorciers alpins, abandonnant leurs corps physiques pour partir vagabonder sous forme d'âme-taon, nous pouvons mettre en évidence une seconde série sémantique qui nous aide, après ce long parcours qui nous a amené jusqu'aux hauts pâturages pour voir les courses des vaches tourmentées par des taons piqueurs, à aborder un modèle d'interprétation pour les déplacements alpins hors-du-corps. Les parlers francoprovençaux de la Vallée d'Aoste nous ont transmis le terme tavané avec une référence intéressante au rêve, dans le sens précis d'errer. Encore une fois, la folkloristique nous permet de suivre la trace de ces voyages aériens jusqu'aux Alpes carniques du Frioul du XVI ${ }^{\mathrm{e}}$ siècle où Carlo Ginzburg (1980) nous raconte des batailles nocturnes des

benandanti,

partant, les nuits des Quatre-Temps, combattre, avec leurs gourdins de fenouil, les sorciers gâteurs de récoltes. Ainsi, la femme d'un des benandanti, Paolo Gasparutto, se rappelle :

[...] qu'une nuit d'hiver, se réveillant effrayée, elle avait appelé Paolo pour qu'il la rassure : «Bien que je l'aie appelé une dizaine de fois en le secouant, je n'ai jamais pu le réveiller et il se tenait le visage tourné vers le haut. » Un peu plus tard, elle l'avait trouvé en train de marmonner : « Ces benandanti disent que lorsqu'ils quittent le corps leur esprit ressemble à une petite souris, de même lorsqu'il revient, et si le corps, pendant qu'il est privé d'esprit était retourné, il demeurerait mort car l'esprit ne pourrait rentrer. » (Ginzburg, 1980 : 40-41 ; italiques mis par nos soins.)

Ce type d'expérience non isolée, vécue et transmise par Gasparutto, avec l'esprit sortant sous forme de petite souris pour un voyage extracorporel, peut être rattaché à une forme plus typiquement sorcellaire, collectée dans les Alpes occidentales, qui répond, comme nous l'avons déjà noté, au motif narratif G251.1 Witch recognized by seeing wasp (beetle) enter her mouth while asleep.

Only when it enters can she be awakened. Ces documents mettent en évidence un processus de métamorphose de l'âme en insecte harceleur ou autres petits animaux nuisibles qui rend compte de la capacité de certaines personnes exceptionnelles de quitter leur corps physique pour errer sous forme d'esprit. De tels récits décrivent clairement une expérience hors-du-corps : ces sujets s'étendent, le plus souvent en position supine, et ils laissent leur esprit, souvent sous forme animale, sortir de leur bouche et revenir, après le vagabondage nocturne, se réintégrer à leur dépouille corporelle, immobile - voire paralysée - au même endroit où ils l'avaient abandonnée.

Ainsi, certaines formes signifiant "rêver ", comme le verbe francoprovençal tavané, transmet une référence matricielle à l'état onirique dans son sens étymologique de " rêver ", soit « errer " (cf. FEW 10, 184-187, * REEXVAGUs). L'analyse de ces matériaux offre une ouverture pour aborder une ethnoscience transculturelle intuitive des insectes (et des autres petits animaux et vermines) afin de tracer leur comportement et 
de mieux mettre en évidence cette âme-insecte revenant de son errance extatique pour réintégrer le corps physique par ses orifices.

Les parlers francoprovençaux de France et de Suisse romande, tout comme les parlers occitans, nous permettent alors d'aborder ce même processus de séparation corpsesprit à partir d'un point de vue différent: les séries verbales du type s'estavani, dérivées de *(EX-) + TABANUS, portent plutôt l'attention sur le corps physique vidé de l'âme partie en errance. La signification de ces verbes nous ramène au champ sémantique de l'évanouissement, de la perte de conscience, comme un corps qui a perdu son esprit, sorti de la bouche sous la forme de notre taon prototypique. Cette famille sémantique regroupant les évanouissements, des sensations d'étouffement et de suffocation, des bourdonnements de tête (FEW 13, 1, 2-3), mais aussi des corps paralysés en position supine et des âmes-taons harcelantes errant dans la nuit, devient, pour nous, une véritable matrice sémantique lexico-narrative originale qui permet de confirmer le lien entre l'imaginaire narratif des voyages extatiques sous forme spirituelle et la base expérientielle de la paralysie du sommeil. Nous pouvons même parler d'une lexie qui a le potentiel d'amorcer une narration plus ou moins développée, à la limite toute compactée dans un seul verbe (p. ex. «(es)tavaner»).

\section{Sémantique de base (core semantics) et adaptations conceptuelles dérivées (conceptual changes) pour les âmes-taons des expériences hors-du-corps en domaine alpin}

20 Cette contribution sur la sémantique des processus de métensomatose de l'âme en insecte diptère s'insère plus largement dans une perspective de sémantique neurocognitive expérientielle que nous avons commencé à esquisser à partir de 2017 (Armand, Cathiard \& Abry, 2017 ; Armand, 2019; Armand, 2020). Une première brique pour la construction d'une telle approche a été posée dans notre dernière contribution publiée dans le Bulletin du Centre d'études francoprovençales (Armand, 2020 : 99-102) où nous avons abordé la construction des catégories terminologiques utilisées transculturellement pour indiquer la phénoménologie du cauchemar, en reconnaissant le rôle fondamental de l'expérience neurocognitive de la paralysie du sommeil - et de son riche sensorium neural - dans les processus de dénomination des êtres fantastiques de type incube qui hantent les imaginaires narratifs humains. Afin d'appréhender ces processus de dénomination, nous avons mis en évidence un modèle pour la construction de ces catégories terminologiques qui prévoit l'indication de l'agent et de son prédicat prototypique lié au champ lexical de l'oppression, de l'écrasement et de la pression, éléments implicites de la même matrice sémantique. Le type lexical chauchevieille francoprovençal et occitan, une vieille qui chauche, qui vient peser sur la poitrine du dormeur, en représente un très bon exemple. En analysant la matrice lexico-narrative repérée pour le mot carco-vèllho / carco-vëllho dans les vallées vaudoises du Piémont, Joisten et Abry nous suggèrent une méthodologie opérationnelle, mettant en rapport des expériences individuelles vécues et leurs interprétations intuitives, pour la construction de notre perspective de sémantique neurocognitive expérientielle ${ }^{8}$ :

Il faut donc chercher avant tout la matrice des expériences sur les êtres de récits de croyance, avant d'avoir recours aux mises en formes de ces expériences rendues possibles par les matrices narratives disponibles, comme des instruments plus ou 
moins élaborés, dans telle ou telle société. Les matrices lexicales (narratives) en sont un condensé qui repère l'expérience et amorce ou non un rendu en récit plus ou moins développé. L'enquête est maïeutique pour éviter de se contenter des premières interprétations (gloses), parfois simplement immédiates, des témoins.

$(2009: 317)$

21 Pour mieux encadrer ces réflexions, nous avons construit notre approche en empruntant la terminologie, inspirée de Susan Carey, adoptée récemment dans le recueil de Barner et Scott Baron (2016), soit core knowledge vs conceptual change. Comme nous l'avons démontré, les composantes essentielles (core knowledge) de genèse sémantique endogène pour les catégories terminologiques se référant à l'expérience du cauchemar - pour la dénomination de l'expérience elle-même et pour ses agents personnifiés - reposent typiquement sur les sensations d'immobilité et d'oppression, fondements neuraux issus des composantes fondamentales de la paralysie du sommeil. Ainsi, pour le terme alpin de chauchevieille, « l'étymologie expérientielle endogène, issue du latin CALCARE et sa relation avec les champs lexicaux de l'oppression, de l'écrasement et de la pression, représentent bien des éléments implicites de cette matrice sémantique neurocognitive pour la construction des terminologies du cauchemar dans les parlers francoprovençaux [et occitans]» (Armand, $2020: 102)$.

Notre contribution actuelle veut attirer l'attention sur les changements secondaires (conceptual changes) qui s'opèrent dans le cadre des composantes sémantiques essentielles (core) en ce qui concerne les notions d'esprit-âme et des expériences horsdu-corps. Nous avons constaté que les travaux sur les métaphores du langage, inspirés de l'approche Embodied Cognition soutenue par Lakoff et Johnson (1999), ne permettent pas d'avoir accès à la sémantique de base pour les expériences objet de notre recherche. Selon leur perspective, les processus cognitifs humains reposeraient sur des métaphores conceptuelles qui s'appuient sur un processus d'embodied mapping, visant à établir des correspondances entre un domaine-source connu, provenant de nos expériences sensorielles et corporelles, et un domaine-cible.

Dans Armand, Cathiard et Abry (2017: 120-122), nous avons mis en évidence deux erreurs concernant la sémantique de la notion d'esprit-âme. La première est issue du travail de Thomas Metzinger qui, contribuant au développement d'une neurophilosophie de l'âme, écrit que "prise comme une métaphore ontologique, la phénoménologie des OBEs conduit inévitablement au dualisme, et à l'idée concrète d'un second corps invisible et sans poids, mais étendu dans l'espace » (2003: 55 ; notre traduction et nos italiques). Nous nous sommes interrogés à la fois sur l'idée d'apesanteur - en tant qu'expérience endogène fondamentale reliée aux sensations d'oppression et d'étouffement ressenties par des individus en paralysie du sommeil, interprétées narrativement comme l'effet d'une attaque sorcellaire-, et sur celle d'invisibilité, en tant qu'adaptation du concept à une expérience exogène. En effet, les enquêtes conduites sur nos terrains transalpins nous ont bien montré que, dans les narrativisations d'expériences hors-du-corps, l'âme-esprit du voyageur en extase est souvent représentée sous forme d'un insecte harcelant ou d'autres petits animaux nuisibles. La deuxième critique touche le travail de Šctrkalj Despot, Skrynnikova et Olszewska sur la notion d'âme en croate, russe et polonais : les auteurs notent que « les mots slaves pour âme dérivent du proto-slave *duxa avec le suffixe -j-a (proto-indoeuropéen *dhousia) et la signification est en relation avec l'action de respirer et de souffler, ce qui est commun à plusieurs langages indo-européens d'où la référence au souffle vital, au principe animateur » $(2012: 466)$. Ce que nous avons trouvé surprenant 
est que l'analyse proposée, s'insérant dans le sillon des métaphores du langage dans l'embodied cognition de Lakoff, ne prend pas en considération le riche matériel ethnographique collecté dans les Alpes dinariques, où des vagabondages nocturnes d'esprits-taons sont bien décrits, nous transmettant ainsi la notion d'une âme bien visible et perceptible en forme d'insecte. En conclusion, nous pouvons souligner que notre approche de sémantique neurocognitive expérientielle correspond bien aux deux temps des études réunies en 2016 par David Barner et Andrew Scott Baron. Pour nous, les composantes essentielles (core knowledge) de genèse sémantique endogène pour les différentes catégories cognitives se référant aux expériences hors-du-corps et à ces esprits-âmes en forme d'insecte reposent typiquement sur les fondements neuraux issus des composantes fondamentales de la paralysie du sommeil, générées dans la jonction temporo-pariétale du cerveau humain. Nous reconnaissons ensuite des interprétations secondaires intuitives, riches en métaphores, de ces expériences endogénérées : il s'agit d'adaptations conceptuelles dérivées (conceptual change), sous influence exogène, qui contribuent à enrichir le sensorium cérébral auquel puisent les imaginaires narratifs que nous avons présentés. Ces représentations intuitives (narrativisation, métaphores, etc.) de l'expérience «core », de base (issue du domaine des contre-intuitions scientifiques), seraient des variations conceptuelles permettant aux sujets de se représenter intuitivement les expériences « core » autrement inaccessibles.

Pour avoir accès à la sémantique cognitive - à ce core meaning - il est nécessaire de développer une perspective transdisciplinaire visant à créer des ponts entre la linguistique, l'ethnographie de la narration et les sciences neurocognitives. En collectant des récits de croyance, domaine privilégié par la folkloristique, nous avons pu ouvrir une voie à la compréhension d'une matrice sémantique pastorale pour des taons qui deviennent des prototypes d'esprits errants et harcelants dans les parlers francoprovençaux et occitans. Le passage en syncope entre ces deux «temps sémantiques " se réalise à travers le "core » des expériences corticales universelles en paralysie du sommeil et leur aménagement par l'expérience mondaine vécue et/ou narrativisée par les sujets. Ainsi nos taons prototypiques, et toutes sortes de petits animaux et vermines, deviennent des outils métaphoriques utiles pour transmettre les représentations intuitives d'âmes-esprits, bien visibles et harassantes, en vagabondage nocturne.

\section{BIBLIOGRAPHIE}

ABRY Christian \& JoISTEN Alice, 2002, « Trois notes sur les fondements du complexe de Primarette. Loups-garous, cauchemars, prédations et graisses ", Le Monde alpin et rhodanien. Revue régionale d'ethnologie, vol. 30, $\mathrm{n}^{\text {os }}$ 1-3 (Le fait du loup. De la peur à la passion : le renversement d'une image), p. 135-161. 
Alinei Mario, 2009, « Da lat. "meridies” “meriggio delle pecore”, a lat. "mora” e lat. "umbra”. Origine italiche e sviluppo linguistico di un termine della pastorizia transumante ", Quaderni di semantica, vol. $30, \mathrm{n}^{\circ} 1$, p. 7-68.

ARMAND Fabio, 2015, «L'espace-temps d'agentivités en sur-intuition dans les narrations des Alpes francoprovençales et de l'Himalaya népalais ", dans C. Dunoyer (éd.), Vivre le temps au jour le jour dans l'aire francoprovençale. Chanter le passé, se projeter dans l'avenir, Actes de la conférence annuelle du Centre d'études francoprovençales (Saint-Nicolas, 29 novembre 2014), p. 15-42.

ARMAND Fabio, 2019, « Kancho bāyu et pako bāyu : esprits en transformation entre crudité et cuisson. Une sémantique exogène pour des rituels funéraires inassouvis au Népal central », dans

F. Armand et C. Dunoyer (éds), Nourrir la mort : rituels funéraires et pratiques alimentaires des Alpes à l'Himalaya, Actes du colloque du Centre d'études francoprovençales (Saint-Nicolas, 27 octobre 2018), p. 81-94.

ARMAND Fabio, 2020, «Une matrice sémantique neurocognitive pour les dénominations du cauchemar dans quelques parlers francoprovençaux de la Vallée d'Aoste », Bulletin du Centre d'études francoprovençales « René Willien », vol. 76, p. 83-106.

ARMAND Fabio, CATHIARD Marie-Agnès \& ABRY Christian, 2016, « Death Divination within a NonDelusional Myth: The Procession of the Dead from the Alps to Himalayas... When a Theoria of "Phantom-Bodies" Meets Its Neural Veridiction Theory ", TricTrac: Journal of World Mythology and Folklore, vol. 9, p. 1-28.

ARMAND Fabio, CATHIARD Marie-Agnès \& ABRY Christian, 2017, « Beyond Nationalistic Isolationisms: Our Species' Brain Dreams and Nightmares. Re-embodying Daemonic Ontologies in the Mediterranean South of the Alps for this 21th Century », dans K. Jurčević Katica, L. Kaliterna Lipovčan et $\mathrm{O}$. Ramljak (éds), Imaging the Mediterranean: Challenges and Perspectives, Institute of Social Sciences Ivo Pilar et VERN Group, p. 113-125.

ARMAND Fabio, CATHIARD Marie-Agnès \& ABRY Christian, 2019, « Neuronuminous-like Experiences within the BRAINCUBUS Framework for Cognitive Folkloristics: Present Promise and Limits », dans Numinoses Erzählen: Das Andere - Jenseitige - Zauberische, Proceedings of Deutsche Gesellschaft für Volkskunde (7-10 septembre 2016), p. 57-65.

ARMAND Fabio \& DUNOYER Christiane, 2019, «Visions de la langue dans le Nouveau dictionnaire de patois valdôtain », dans R. Vautherin, Supplément au Nouveau dictionnaire de patois valdôtain, Centre d'études francoprovençales « René Willien », Aoste, Tipografia Duc, p. 7-17.

ALF = GILLIÉRON Jules \& EDMONT Edmond, 1902-1910, Atlas linguistique de la France, Paris, Éditions Champion.

ALLy = GARDETTE Pierre, 1952-1976, Atlas linguistique et ethnographique du Lyonnais, Paris, Éditions du CNRS.

ALMC = NAUTON Pierre, 1952-1961, Atlas linguistique et ethnographique du Massif central, Paris, Éditions du CNRS.

ATU = UTHER Hans-Jürg, 2011, The Types of International Folktales. A Classification and Bibliography, 3 vol., Folklore Fellow Communications, $\mathrm{n}^{\text {os }}$ 284-286.

BARNER David \& SCOTT BARON Andrew, 2016, Core Knowledge and Conceptual Change, New York, Oxford University Press.

BlAnKe Olaf, ORTIGUe Stephanie, LANDIS Theodor \& SEECK Margitta, 2002, « Stimulating Illusory Own-Body Perceptions », Nature, vol. 419, p. 269-270. 
BONNET Marie, 1994, Tradizioni orali delle valli valdesi del Piemonte [1910-1914], Turin, Claudiana.

CATHIARD Marie-Agnès \& ARMAND Fabio, 2014, « BRAINCUBUS : vers un modèle anthropologique neurocognitif transculturel pour les "fantômes" de l'imaginaire ", dans P. Pajon et M.-A. Cathiard (éds), Les imaginaires du cerveau, Fernelmont, Intercommunications \& Éditions modulaires européennes, p. 53-87.

CERLOGNE Jean-Baptiste, 1907, Dictionnaire du patois valdôtain précédé de la petite grammaire du dialecte valdôtain, Aoste, Imprimerie catholique.

CHENAL Aimé \& VAUTHERIN Raymond, 1997, Nouveau dictionnaire du patois valdotain, Aoste, Musumeci éditeur.

DURAFFOUR Antonin, 1969, Glossaire des patois francoprovençaux, Paris, Éditions du CNRS.

FEW = WARTBURG Walther VON (éd.), 1928-, Französisches etymologisches Wörterbuch: eine Darstellung des galloromanischen Sprachschatzes, Bâle, R. G. Zbinden.

GINZBURG Carlo, 1980, Les batailles nocturnes : sorcellerie et rituels agraires en Frioul, XVI-XVII ${ }^{e}$ siècle, Verdier [I Benandanti: Stregoneria e culti agrari tra Cinquecento e Seicento, Turin, Einaudi, 1972,

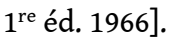

GinZBURG Carlo, 1989, Storia notturna, Turin, Einaudi [Le sabbat des sorcières, Paris, Gallimard, 1992]. GLAREY Miranda, 2011, Dictionnaire du patois de Champorcher, Saint-Christophe, Tipografia Duc. GPSR = GAUCHAT Louis, JEANJAQUET Jules \& TAPPOLET Ernest, 1934-, Glossaire des patois de la Suisse romande, Neuchâtel / Paris, Éditions V. Attinger.

GRUPPO « AMIS DU PATOIS », 2007, Dizionario del dialetto francoprovenzale di Hône, Aoste, Le Château Edizioni.

GUICHARDAZ Celestino \& FASsò Andrea, 1974, La parlata francoprovenzale di Cogne. Il lessico rustico, Turin, Giappichelli editore.

JALLA Jean, 1926, Légendes des vallées vaudoises, Torre Pellice.

JOISTEN Alice \& ABRY Christian, 2009, « Du Bâtoun dë Jacoub à la Carco-vëllho des vallées des Vaudois du Piémont ou des matrices lexicales et narratives à la matrice des êtres de récits de croyances? ", dans D. Jalla (éd.), Héritage(s). Formazione e trasmissione del patrimonio culturale valdese, Turin, Claudiana, « Collana della Società di studi valdesi », $n^{\circ} 28$, p. 311-332.

JoISTEN Charles, 2005-2010, Êtres fantastiques du Dauphiné, vol. I : Patrimoine narratif de l'Isère (2005, 576 p.) ; vol. II : Patrimoine narratif des Hautes-Alpes (2006, 608 p.) ; vol. III : Patrimoine narratif de la Drôme (2007, 256 p.). - Êtres fantastiques de Savoie, vol. I : Patrimoine narratif du département de la Savoie (2009, 560 p.) ; vol. II : Patrimoine narratif de la Haute-Savoie (2010, 512 p.), édition préparée par A. Joisten et N. Abry, Grenoble, Musée dauphinois.

LAKOFF George \& JoHnson Mark, 1999, Philosophy in the Flesh, New York, Basic Books.

LAVOYER Ivo, 2017, Dictionnaire du patois de Chambave, Saint-Christophe, Tipografia Duc.

MeTZINGER Thomas, 2003, Being No One, Cambridge, MIT Press.

MOTTE-FLORAC Élisabeth, \& THOMAS Jacqueline M. C., 2003, Les « insectes » dans la tradition orale, Paris, Peeters.

ŠctrKalj Despot Kristina, SKRYNNIKova Inna \& OlszewSKA Julia Ostanina, 2012, « Cross-Linguistic Analysis of Metaphorical Conceptions of душА / dusza / duša ('soul') in Slavic Languages 
(Russian, Polish, and Croatian) », dans Proceedings of the 38th Annual Meeting of the Berkeley

Linguistics Society, Berkeley, University of California, p. 465-480.

THOMPSON Stith, 1966, Motif-Index of Folk-Literature, éd. révisée et augmentée, Bloomington /

Londres, Indiana University Press.

\section{NOTES}

1. Dans les conclusions de sa Storia notturna, Carlo Ginzburg (1989: 289, note 3) renvoie aux collectes réalisées par Jean Jalla et Marie Bonnet, en indiquant que «il calabrone (galabroun) è detto masc, "strega" ".

2. Les insectes sortant des bouches des autres sorciers vaudois sont une grosse mouche (Massel) et un bourdon (Villar Pellice). Intéressante à constater, dans la version de Pomaret, la présence d'un papillon entrant dans la bouche de la jeune fille ensorcelée : « La jeune fille entrouvre ses lèvres en un gracieux sourire, et un papillon qui volait, depuis quelques instants, autour de sa tête, entre dans sa bouche, et n'en ressort plus. Au même instant, la paysanne s'étire et ouvre ses yeux tout larges. En voyant le jeune homme, elle rougit violemment et se lève d'un bond, honteuse de s'être laissée surprendre étendue. Mais aussitôt après, une ombre s'étend sur son visage, et elle fixe le jeune homme dans le blanc des yeux, en lui demandant: "Y-a-t-il longtemps que vous êtes entré ?" "Je ne sais pas. Mais, dites-moi, vous aimez donc avaler des papillons ?" La jeune fille répond avec angoisse: "Oh! Je sais bien, vous ne m'aimerez plus, maintenant! Vous ne voudrez plus de moi! Mais du moins, jurez-moi que vous ne direz à personne ce que vous avez vu !" (La jeune fille, parait-il, n'appartenait pas à une famille de sorciers, mais elle avait été ensorcelée par un mauvais individu, et ne pouvait se délivrer du « sort » qui lui avait été imposé.) 》

3. Nous rappelons aussi la référence au récit-type ATU $1635 \mathrm{~A}$ - Dream of Treasure Bought (Guntram), auquel appartient le rêve du roi de Bourgogne, Gontran (561-592), documenté pour la première fois en Europe dans l'Historia Langobardorum (datée de 784 ou, au plus tard, 789) de Paul Diacre (voir Armand, 2015).

4. L'aire où l'on trouve des formes dérivée de *EX- + TABANUS comprend deux points dans le Puyde-Dôme (47, Valcivière ; 57, Saint-Anthème) et dans la Loire (48, Essertines-en-Chatelneuf ; 58, Usson).

5. Consultable en ligne sur le site < www.ieo12.org/d7/recerca-diccionari-cantalausa> sous l'entrée estavanir [consulté le 28 octobre 2020].

6. Consultable en ligne sur le site <www.etymologie-occitane.fr> sous l'entrée tavan [consulté le 28 octobre 2020].

7. Consultable en ligne sur le site < www.etymologie-occitane.fr//?s=tavan> [consulté le 28 octobre 2020].

8. Nous renvoyons au cadre heuristique permettant de rechercher les intuitions, contre-intuitions et sur-intuitions dont relèvent les informations élicitées par notre approche d'anthropologie et de sémantique neurocognitives: voir Armand (2015); pour une version en anglais, voir Armand, Cathiard \& Abry (2016). 


\section{RÉSUMÉS}

Dans le cadre du développement d'une perspective de sémantique neurocognitive expérientielle, nous nous intéresserons aux processus de métensomatose de l'âme en insecte. Nous conduirons nos réflexions en partant des formes tavané, dérivés du latin TABANUs, des variétés francoprovençales de la Vallée d'Aoste, des développements francoprovençaux de France et de Suisse romande et de ceux occitans de la série verbale formée du composé $*_{\text {EX- }}+$ TABANUS, pour aboutir à la reconstruction d'une matrice sémantique lexico-narrative qui décrit les bases expérientielles de la paralysie du sommeil (paralysie, sensations d'étouffement et suffocation, bourdonnement de tête, etc.). En empruntant la terminologie adoptée dans le recueil de Barner et Scott Baron (2016), soit core knowledge vs conceptual change, nous focaliserons notre attention sur les changements secondaires (conceptual changes) qui s'opèrent dans le cadre des composantes sémantiques essentielles pour ce qui concerne les notions d'âme-esprit et des expériences hors-du-corps.

Within the context of the development of an experiential neurocognitive semantic perspective, we investigate the processes of metensomatosis of the soul as an insect. We will conduct our reflections starting from the forms tavané, derived from the Latin TABANUS, in the Francoprovençal varieties of Aosta Valley, the Francoprovençal developments of France and Roman Switzerland and the Occitan ones of the verbal series formed by the compound * EX- + TABANUS, in order to reconstruct a lexico-narrative semantic matrix that describes the experiential bases of sleep paralysis (paralysis, sensations of choking and suffocation, head buzzing, etc.). Byborrowing the terminology adopted in Barner and Scott Baron's publication (2016), core knowledge vs conceptual change, we will focus mainly on the secondary changes (conceptual changes) that take place within the framework of the core semantic components of mind-soul concepts and out-of-body experiences.

\section{INDEX}

Keywords : experiential neurocognitive semantics, core knowledge vs conceptual change, sleep paralysis, soul-horsefly, Francoprovençal, Occitan

Mots-clés : sémantique neurocognitive expérientielle, core knowledge vs conceptual change, paralysie du sommeil, esprit-taon, francoprovençal, occitan

\section{AUTEUR}

\section{FABIO ARMAND}

Institut Pierre Gardette, Pôle « Culture(s), Langue, Imaginaires », UR Confluences : Sciences et Humanités, Université catholique de Lyon (UCLy)

armand.fabio@gmail.com 